\title{
Growing a garden of neurons
}

\section{Rebekah C. Evans* and Sridevi Polavaram}

Center for Neural Informatics, Krasnow Institute for Advanced Study, George Mason University, Fairfax, VA, USA

*Correspondence: rcolema2@masonlive.gmu.edu

Edited by:

John Van Horn, University of California at Los Angeles, USA

Keywords: morphology, dendrites, growth algorithm, computational model, neural networks

\section{A commentary on}

Self-referential forces are sufficient to explain different dendritic morphologies by Memelli, H., Torben-Nielsen, B., and Kozloski, J. (2013). Front. Neuroinform. 7:1. doi: 10.3389/fninf.2013.00001

Computational models of biologically realistic neuronal networks have advanced neuroscience in the past 20 years. With an ultimate goal of simulating a whole brain, these networks must become larger and more complex. However, a sheer massive number of neurons do not make a brain. Neurons are all different, with different kinetics, neurotransmitters, and importantly different morphologies. A network can be made by connecting copies of the same cell together, but this kind of homogenous network can only explain so much. Real neuronal networks are heterogeneous and are made up of neurons that follow both intrinsic and extrinsic cues to grow their unique dendritic arbors (Scott and Luo, 2001). In addition to homogenous and heterogeneous network models, hybrid network models have been implemented by creating a small heterogeneous network and replicating it to establish a larger network (Kozloski, 2011). However, modeling studies have shown that homogenous networks act differently than realistic heterogeneous ones (MäkiMarttunen et al., 2011). Because computational neuronal networks need to grow larger to simulate complete brain regions, and because heterogeneity in a network is critical to modeling a realistic brain, algorithms for digitally generating neural morphologies are a necessary step toward this goal.

A new paper by Memelli et al. (2013) joins the field of papers providing algorithms for growing digital neurons. Their algorithm can be used to build a network consisting of millions of neurons each with a unique morphology. The current models, L-Neuron (Ascoli et al., 2001), NeuGen2.0 (Wolf et al., 2013), NetMorph (Koene et al., 2009), and CD3X (Zubler and Douglas, 2009) have made great strides in advancing the process of generating digital neurons. These models are all publicly available, and can be used to generate large networks of neurons. Recently L-Neuron was used to generate a 0.5 million cell model of the dentate gyrus (Schneider et al., 2012). Each algorithm has its own specific advantages. NetMorph has a synapse-generating algorithm, NeuGen2.0 is modular and adaptable to new data, and CD3X can isolate intrinsic and extrinsic factors of neuron development by growing the same neurons in different model environments. In combination with the parallelization of simulation software [such as NEURON (Migliore et al., 2006)], these neuron generators are laying the groundwork for enabling massive biologically realistic simulations.

Memelli et al. (2013) do not attempt to model the molecular mechanisms of dendritic growth, but instead work to make a concise, computationally efficient model that can capture the structure and variability of realistic morphologies. Their work adds two elements to this field. First, it simplifies the neural growth algorithm to contain a combination of three biologically inspired intrinsic parameters: somaoriented tropism, dendritic self-avoidance, and membrane stiffness. The three parameters of their growth algorithm are all intrinsic to the cell itself and do not take into account any extrinsic signals that could come from other neurons or physical constraints. Each of these parameters has been previously described, but Memelli et al. are the first to combine them in one simple model. Second, their algorithm is written to be fast and massively parallel, creating the possibility for generating billions of neurons on the IBM Bluegene computer. Their algorithm can generate a neuron in less than two seconds, and when run on parallel cores is capable of generating enough neurons to simulate an entire brain region. Together, these elements fit the need to have morphological diversity within a network as well as the need to have extremely large networks.

Each of the current morphology simulators has their particular strengths. The ideal situation would be for Memelli's new algorithm to be incorporated into one of the existing ready-to-use packages. For example, the application of this algorithm within the external constraints of CX3D could help isolate the extrinsic and intrinsic aspects of dendritic arborization. When used together these simulators can help create massive-scale heterogeneous networks for computational modelers and can help investigate how dendrites actually grow.

\section{REFERENCES}

Ascoli, G. A., Krichmar, J. L., Scorcioni, R., Nasuto, S. J., and Senft, S. L. (2001). Computer generation and quantitative morphometric analysis of virtual neurons. Anat. Embryol. 204, 283-301. doi: 10.1007/s004290100201

Koene, R. A., Tijms, B., van Hees, P., Postma, F., de Ridder, A., Ramakers, G. J. A., et al. (2009) NETMORPH: a framework for the stochastic generation of large scale neuronal networks with realistic neuron morphologies. Neuroinformatics 7, 195-210. doi: 10.1007/s12021-009-9052-3

Kozloski, J. (2011). Automated reconstruction of neural tissue and the role of large-scale simulation. Neuroinformatics 9, 133-142. doi: 10.1007/s12021011-9114-1

Mäki-Marttunen, T., Aćimović, J., Nykter, M., Kesseli, J., Ruohonen, K., Yli-Harja, O., et al. (2011). Information diversity in structure and dynamics of simulated neuronal networks. Front. Comput. Neurosci. 5:26. doi: 10.3389/fncom.2011. 00026

Memelli, H., Torben-Nielsen, B., and Kozloski, J. (2013). Self-referential forces are sufficient to 
explain different dendritic morphologies. Front. Neuroinform. 7:1. doi: 10.3389/fninf.2013.00001

Migliore, M., Cannia, C., Lytton, W. W., Markram, H., and Hines, M. L. (2006). Parallel network simulations with NEURON. J. Comput. Neurosci. 21, 119-129. doi: 10.1007/s10827-0067949-5

Schneider, C. J., Bezaire, M., and Soltesz, I. (2012). Toward a full-scale computational model of the rat dentate gyrus. Front. Neural Circuits 6:83. doi: 10.3389/fncir.2012.00083

Scott, E. K., and Luo, L. (2001). How do dendrites take their shape. Nat. Neurosci. 4, 359-365. doi: $10.1038 / 86006$
Wolf, S., Grein, S., and Queisser, G. (2013). Employing NeuGen 2.0 to automatically generate realistic morphologies of hippocampal neurons and neural networks in 3D. Neuroinformatics 11, 137-148. doi: 10.1007/ s12021-012-9170-1

Zubler, F., and Douglas, R. (2009). A framework for modeling the growth and development of neurons and networks. Front. Comput. Neurosci. 3:25. doi: 10.3389/neuro.10.025. 2009

Received: 02 August 2013; accepted: 02 August 2013; published online: 26 August 2013.
Citation: Evans RC and Polavaram S (2013) Growing a garden of neurons. Front. Neuroinform. 7:17. doi: 10.3389/fninf.2013.00017

This article was submitted to the journal Frontiers in Neuroinformatics.

Copyright (c) 2013 Evans and Polavaram. This is an open-access article distributed under the terms of the Creative Commons Attribution License (CC BY). The use, distribution or reproduction in other forums is permitted, provided the original author(s) or licensor are credited and that the original publication in this journal is cited, in accordance with accepted academic practice. No use, distribution or reproduction is permitted which does not comply with these terms. 